\title{
Editorial. Militaires et engagements extérieurs : à la conquête des cœurs et des esprits?
}

Editorial : Soldiers and external deployments : To conquer Hearts and Minds?

\section{Christian Olsson et Pauline Vermeren}

\section{(2) OpenEdition}

\section{Journals}

Édition électronique

URL : http://journals.openedition.org/conflits/3109

DOI : $10.4000 /$ conflits.3109

ISSN : $1777-5345$

Éditeur :

CCLS - Centre d'études sur les conflits lilberté et sécurité, L'Harmattan

Édition imprimée

Date de publication : 15 novembre 2007

Pagination : 7-12

ISBN : 978-2-296-04582-8

ISSN : 1157-996X

Référence électronique

Christian Olsson et Pauline Vermeren, «Editorial. Militaires et engagements extérieurs : à la conquête des cœurs et des esprits? », Cultures \& Conflits [En ligne], 67 | automne 2007, mis en ligne le 20 février 2008, consulté le 30 mars 2021. URL : http://journals.openedition.org/conflits/3109 ; DOI : https:// doi.org/10.4000/conflits.3109 


\title{
Editorial.
}

Militaires et engagements extérieurs :
à la conquête des cœurs et des esprits ?

\section{Christian OLSSON}

\section{Pauline VERMEREN}

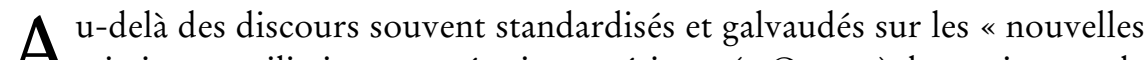

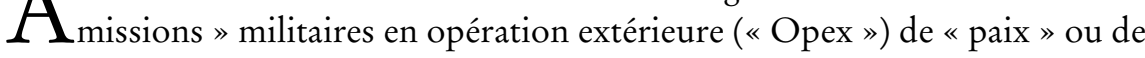
"stabilisation », ce numéro de Cultures $E$ Conflits se propose de déplacer le regard sur ces pratiques internationales de coercition pour les éclairer par un certain nombre de problématiques historiques, politiques et sociologiques plus larges. Ce « décentrement » du regard découle ici de trois constats.
\end{abstract}

Premièrement, toute réflexion sur les Opex doit s'engager en amont mais aussi en aval - des engagements militaires extérieurs et des formes de conflictualité qui les justifient ou qui sont évoquées pour les justifier. En effet, les ramifications empiriques de cet objet flou sont à la fois plus complexes et multiples. De ce point de vue, différentes focales et sites empiriques sont ici explorés : les doctrines militaires, les technologies de coercition, les relations organisationnelles et inter-organisationnelles au sein du monde des professionnels de la sécurité, les évolutions sociétales plus larges au sein des Etats intervenants, les modes d'interaction avec les populations locales, etc. Cela nous conduira parfois à aborder l'objet par des biais, ou même des tangentes, qui pourraient sembler contre-intuitifs au premier abord.

En effet, deuxièmement, si l'on ne veut pas se contenter de reproduire les découpages opérés par le langage politique contemporain - au demeurant très imprécis lorsqu'il s'agit de définir les « interventions », les Opex ou les "projections de force » de l'après-Guerre froide - il faut (re)construire l'objet au travers d'une problématisation plus large, impliquant parfois de sortir des «sentiers battus » des débats politiques et académiques traditionnels. Ces derniers sont en effet souvent structurés et limités par les distinctions propres aux politiques gouvernementales (politique intérieure / politique étrangère) et aux approches disciplinaires (science politique «interniste »/ relations internatio- 
nales). La perspective d'analyse se veut par conséquent ici résolument interdisciplinaire, mobilisant notamment les apports de la sociologie, de l'histoire et de la science politique, mais aussi de la socio-histoire et de la théorie politique.

Troisièmement, dans un contexte dans lequel « l'interopérationalité » est le credo sacro-saint de la majorité des institutions militaires « occidentales », il va quasiment de soi de s'intéresser à des cas «nationaux » divers, voire de les comparer. Mais généralement, le retour du refoulé du «nationalisme méthodologique » se fait alors souvent par le biais de la perspective «nationale », le regard structuré par les enjeux nationaux du moment, porté sur ces cas multinationaux. Soucieux d'éviter cet écueil, ce numéro s'inscrit notamment dans le cadre d'une collaboration internationale plus large et plus ancienne entre Cultures $\mathcal{E}$ Conflits et la revue italienne Conflitti Globali dont nous avons dans ce numéro l'honneur de publier trois contributeurs : Massimiliano Guareschi, Maurizio Guerri et Salvatore Palidda.

Au-delà de la diversité des perspectives et des "terrains », diversité qui comporte inévitablement des lacunes (dont le cas des Opex sur le continent africain n'est pas des moindres), les articles de ce numéro se situent à l'interface de trois problématiques, trois lignes de force transversales et interdépendantes relatives aux transformations des pratiques étatiques de coercition.

La première est celle de la légitimation de l'usage de la force militaire par-delà les frontières et notamment auprès des populations locales sur les théâtres de déploiement des armées. En effet, au-delà d'une approche statique en termes d'un «monopole de la violence légitime » dont bénéficierait la «force » militaire face à une «violence " protéiforme émanant des acteurs politiques «locaux », il faut aborder la légitimité non pas comme monopole ou comme attribut, mais à la fois comme processus et comme relation, c'est-à-dire comme légitimation. Ainsi Thomas Lindemann et Christian Olsson, malgré des différences de perspectives, insistent tous deux sur l'impératif de légitimation « locale » du recours à la force dans le cadre d'opérations militaires ayant la prétention de participer de la «pacification » de relations sociales conflictuelles. Ils montrent également la difficulté que cet impératif représente pour des armées habituées - notamment de par leur socialisation aux valeurs de la « guerre conventionnelle » - à considérer la « violence légitime » (mais légitime pour qui ?) comme un attribut consubstantiel à leur statut d'institution étatique et donc comme transposable au contexte des Opex contemporaines souvent caractérisées par l'omniprésence de formes non étatiques de violence. Cependant, au-delà des seules populations locales, les relations de légitimation / dé-légitimation du recours à la force impliquent en réalité une multiplicité d'audiences et de communautés interprétatives d'une telle légitimité.

Parmi celles-ci, et ce dans un contexte où les Opex sont souvent perçues comme « guerres par choix », à défaut de pouvoir être perçues comme « guerres par nécessité » (malgré l'énonciation des menaces à la sécurité nationale qui 
les accompagne généralement), on ne saurait sous-estimer les audiences internes aux Etats intervenants. Ainsi, Jean-Paul Hanon montre notamment comment la conception des « projections de force » allemandes, et notamment leur subordination aux architectures policières, procède d'un impératif (non exempt du poids de l'histoire) de « crédibilisation » de celles-ci à la fois auprès des citoyens allemands et des étrangers sur le territoire allemand. On observe alors comment le discours sur les " cœurs et les esprits ", dont la genèse historique est inséparable de pratiques de légitimation déployées dans l'environnement immédiat des opérations militaires, désigne désormais souvent des audiences « externes » d'une part, le «front intérieur » (l'opinion publique à l'intérieur de l'Etat intervenant) d'autre part, au point que les différentes logiques sont de plus en plus difficiles à désenchevêtrer.

La deuxième ligne de force relève ainsi de l'analyse des relations entre logique policière et logique militaire, entre sécurité intérieure et sécurité extérieure au sens traditionnel de ces termes, dans des missions qui sont pourtant menées « en externe ». La question du rapport entre ces deux logiques - si tant est qu'on puisse parler de logiques - se trouve en effet au cour des Opex contemporaines. Elle est ici abordée d'au moins trois points de vue distincts, mais non mutuellement exclusifs les uns des autres.

Le premier consiste à s'intéresser à la manière dont des modes de raisonnement, des technologies de coercition et de contrôle ainsi que des pratiques routinières traditionnellement considérées comme policières, pénètrent dans les champs de pratique militaire - et cela bien au-delà des seules missions de police militaire ou de gendarmerie. Georges-Henri Bricet des Vallons montre ainsi comment la stratégie américaine de « l'arme non létale », et les technologies et imaginaires qui la sous-tendent, casse la distinction entre logique policière et logique militaire, entre civil et militaire, entre maintien de l'ordre et maintien de la paix, et importe cette logique «globale» dans une stratégie militaire nourrissant et se nourrissant d'un certain nombre de concepts développés en rapport aux Opex américaines, que ce soit celui de Low Intensity Conflicts (LIC, conflits de basse intensité), de Military Operations Other Than War (MOOTW, opérations militaires autres que la guerre), ou encore de contre-insurrection.

Le deuxième point de vue consiste à analyser la façon dont les relations entre polices et militaires dans le cadre des " projections de force », mais aussi en amont et en aval de celles-ci, se transforment et reconfigurent les interactions entre les champs de pratiques respectifs. Jean-Paul Hanon analyse ainsi en détail le phénomène de prédominance graduelle des systèmes de police sur les architectures militaires dans le cas des «interventions » allemandes. Quelles en sont les causes et les conséquences ? Comment reconfigure-t-il les luttes bureaucratiques au sein du monde des professionnels de la sécurité ? Il s'agit en effet d'une problématique cruciale qui touche à la nature même des changements histori- 
ques - en dépit des continuités - de la structure des missions « extérieures ». Elle semble en effet ne pas concerner qu'une armée nationale particulière ou un type de mission spécifique. Elle affecte au contraire l'ensemble des Opex contemporaines. De ce point de vue, le « retour » et la diffusion transnationale des doctrines de la contre-insurrection britanniques et américaines d'une part et la « policiarisation » des missions de « gestion de crise »-ici analysée à travers les cas allemand et italien - d'autre part, ne sont que les deux faces, très différentes au demeurant, d'un phénomène plus général d’interpénétration des logiques de sécurité intérieure et de sécurité extérieure.

Le troisième point de vue consiste à s'intéresser aux logiques de « retour sur sécurité intérieure » par lesquelles certaines pratiques militaires développées dans le cadre des missions à l'étranger sont partiellement réimportées en interne, pour souvent être ré-exportées en externe ensuite. C'est notamment ce jeu de «va-et-vient» que met en évidence Salvatore Palidda à propos des carabineri italiens, tout en analysant certains des ressorts politiques et sociaux de l'engagement italien dans les «nouvelles Opex ». Mathieu Rigouste, dont l'article ne porte pas sur les Opex, montre à ce propos que l'intérêt au sein de certaines sphères militaires pour des questions traditionnellement considérées comme de « sécurité intérieure " n'est pas un phénomène nouveau, mais relevait jusque là essentiellement de la réimportation partielle de savoir-faire et de doctrines coloniaux développés hors de la « métropole ».

Il faut cependant se garder de considérer « l'armée », "l'institution militaire » ou «les militaires » comme des entités homogènes et/ou unifiées conduisant à réfléchir exclusivement en termes de traditions militaires nationales. L'hétérogénéité des processus de formation des identités militaires au sein des appareils militaires nationaux et de plus en plus - interopérationnalité oblige - multinationaux, implique notamment de voir que cette « policiarisation » des militaires n'est que relative. Elle est l'objet de nombreuses luttes et de résistances au sein même des institutions et des bureaucraties militaires. Cela est d'autant plus le cas que l'indistinction entre ce qui relève - conformément à la différentiation propre à l'Etat-nation moderne - des domaines réservés de la police d'une part (maintien de l'ordre et de la paix civile) et des militaires d'autre part (la guerre), se fait bien souvent au profit des forces de coercition intermédiaires comme pour la France la gendarmerie voire, dans une moindre mesure, les troupes de marine et, dans le cas de l'Italie, les carabinieri.

La troisième ligne de force relève de l'histoire et de la socio-histoire. Si les contributeurs de ce numéro s'intéressent en priorité à l'analyse de missions militaires récentes ou actuelles, la démarche de ce numéro - en refusant de se laisser aveugler par le discours sur la nouveauté radicale - est également historique. Il s'agit d'analyser les doctrines militaires contemporaines de recours à la force et de «pacification de populations » du point de vue de leur genèse historique, cela en refusant la césure simple de la fin de la Guerre froide ou, 
plus récemment encore, de la « guerre globale contre le terrorisme ». En effet, pour peu que l'on veuille bien s'intéresser à la fois aux continuités et aux changements historiques, de nouvelles perspectives s'ouvrent. Ainsi, les «nouvelles opérations de paix » ou de «stabilisation » semblent, au-delà des débats doctrinaux foisonnants auxquels ils ont donné lieu dans les années 1990, prolonger dans une certaine mesure des trajectoires historiques plus anciennes. Ainsi, de la même façon que les théories de la «non-létalité » et du "contrôle des foules » s'inscrivent dans une histoire américaine des années 1960 et 1970 pourtant encore fortement marquée par la distinction interne/ externe (Georges-Henri Bricet des Vallons), les doctrines de la contre-insurrection des années 1960 et 1970 semblent inscrire les «nouvelles » opérations de stabilisation - notamment en Afghanistan ou en Irak - dans des histoires plus longues (Christian Olsson). Mais s'agit-il de continuités historiques malgré les lignes d'hétérogénéité, d'une résurgence après la "parenthèse » de l'aprèsVietnam, voire après les LIC des années 1980 (pour le cas américain) ou de l'après-Algérie (pour le cas français) ou de la « réinvention d'une tradition » ? Seule une approche socio-historique peut permettre de répondre à cette question. De ce point de vue, alors que les théories de la « guerre révolutionnaire » sont réactualisées par la doctrine militaire américaine, l'article de Mathieu Rigouste montre que celles-ci n'ont pas pour autant disparu du paysage français après la guerre d'Algérie, mais qu'elles ont simplement subi des transformations, notamment en venant nourrir un réflexion stratégique militaro-policière sur le « contrôle sécuritaire ».

$\mathrm{Au}$ travers de ces trois problématiques, ces trois lignes de force, l'ambition est de renouveler l'analyse des pratiques militaires contemporaines - ne seraitce qu'à la marge - en cassant les distinctions simples au travers desquels on les aborde souvent : les catégories fixistes du légitime (l'« humanitaire », la « responsabilité de protéger », etc.) et de l'illégitime (le «terrorisme », mais aussi la guerre «d'agression », etc.) ne tenant pas compte des processus de (dé-)légitimation qui les sous-tendent, les sphères strictement délimitées de l'interne (police) et de l'externe (militaire) et, enfin, la distinction trop claire entre le nouveau et l'ancien.

Il faut alors, comme le font Massimiliano Guareschi et Maurizio Guerri, replacer les évolutions contemporaines dans une réflexion plus générale sur les transformations historiques multiples de la «forme-guerre ». En effet, il faut comprendre comment la catégorie générique de « guerre », par ailleurs bannie des discours politiques sur les engagements militaires extérieurs contemporains, se transforme. De ce point de vue, l'analyse des idéaux régulateurs des " conflits de projection» contemporains, celle de l'" humanité » (et/ou de l'humanitaire), de la «paix » ou moins ambitieusement de la «stabilité »idéaux par définition en décalage par rapport à la réalité d'un certain nombre de pratiques militaires - ouvre sur une question plus générale mais aussi plus dérangeante : dans quelle mesure les engagements militaires extérieurs et leurs 
figures propres du combattant ne sont-ils pas révélatrices de la métamorphose de la forme-guerre contemporaine, plutôt que d'une distinction stricte entre une guerre devenue illégale (sauf exception) d'une part, et les formes et les modes d'action militaires en «Opex» d'autre part ? 